\title{
Éditorial
}

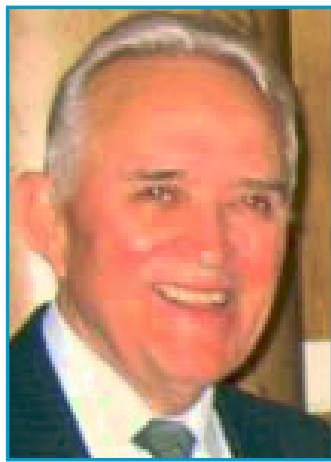

\section{La SHF : Pourquoi ? Pour qui ? Comment ?}

\author{
Pourquoi ?
}

La mission fondamentale de la SHF est de recueillir, valoriser et diffuser des connaissances scientifiques et techniques dans les domaines de l'eau : hydrologie, hydraulique, mécanique des fluides, énergie, aménagements, transport fluvial et maritime, environnement...

D'où son caractère pluridisciplinaire.

Les connaissances constituent aujourd'hui une ressource importante, immatérielle, qui ne se cède pas mais se partage ; $d^{\prime}$ aucuns pensent même que le $X X I^{e}$ siècle sera celui de la connaissance.

Pour qui ?

La SHF accueille tous les acteurs concernés par les domaines de l'eau : chercheurs, universitaires, étudiants, ingénieurs, gestionnaires, membres d'entreprises, de bureaux d'études, de laboratoires, d'organismes publics et privés, ...

D’où son caractère interprofessionnel.

\section{Comment?}

La SHF a créé un organisme original, le Comité Scientifique et Technique (CST) qui regroupe quelque 300 membres. Le CST constitue des réseaux fédérant les compétences, assurant un rôle de passeur. Ce « cerveau collectif » pense plutôt en français et permet de réussir une synergie intellectuelle et culturelle efficace.

Le travail s'effectue dans trois divisions regroupant les différentes disciplines:

- Mécanique des fluides : circuits, machines, cavitation, écoulements polyphasiques, microfluidique, ...

- Eau et environnement : quantité et qualités des ressources, hydrologie (y compris hydro-météorologie), phénomènes naturels (crues et inondations, sécheresses) et leurs conséquences, transports solides), protection de l'environnement, gestion économique et sociale, ...

- Hydraulique fluviale et maritime : navigation, canaux, ports, aménagements fluviaux et maritimes, ...

Toutes ces disciplines utilisent largement des outils de modélisation de plus en plus sophistiqués qui aident à la compréhension de phénomènes et contribuent largement à l'aide à la décision.

Une fois les connaissances recueillies et valorisées, elles sont diffusées par trois canaux principaux:

- Les manifestations (séminaires, colloques et congrès) qui rassemblent sur un à trois jours tous les acteurs intéressés par le thème. La SHF organise 4 à 5 manifestations par an. C'est l'occasion d'échanges avec des collègues confrontés aux mêmes problèmes.

- La revue « La Houille Blanche », seule revue internationale de l'eau francophone.

- Le site Internet, sur lequel on retrouve maintenant les articles publiés dans «La Houille Blanche ». Cet « outil de travail interactif » vous sera présenté, en détail, par André Daubert, vice-président du CST, dans le prochain numéro de « La Houille Blanche».

La SHF travaille en collaboration avec d'autres associations. Dans le domaine international, la SHF a établi un partenariat avec l'AIRH, et elle est membre du Conseil Mondial de l'Eau.

La SHF a entrepris une réforme pour s'adapter aux attentes de ses membres.

Donc, avec le dynamisme de tous ses membres, la SHF a de beaux jours devant elle.

Yves Marolleau

Président du Comité scientifique et technique

PS : Le $1^{\text {er }}$ juillet 2007, après deux quinquennats, je quitterai mon poste. Pierre-Louis Viollet qui me remplacera, s'exprimera très prochainement dans cette revue. Je resterai au service de notre Société. 